\title{
Impregnación superficial, con azufre, del hormigón
}

\author{
F. DUTRUEL, J P. LEHAIR \\ Centro de estudios e investigaciones de la industria del hormigón manufacturado \\ Betons Industriels*, N.o 54-2.० trimestre 1976
}

Se puede afirmar que la durabilidad del hormigón está principalmente condicionada a su compacidad y a su aislamiento de los ambientes agresivos. Los agentes destructores endógenos se consideran eliminados cuando el hormigón está preparado correctamente, con materiales durables y técnica perfecta de amasado, colocación y curado.

El centro francés, de estudios e investigaciones de la industria del hormigón "manufacturado" ha emprendido un estudio experimental de la impregnación con azufre. El principal objetivo ha sido comprobar la persistencia, a largo plazo, de las mejoras que se alcanzan en las propiedades físicas y químicas del hormigón por el revestimiento, o mejor, impregnación a cierta profundidad con azufre.

\section{INTRODUCCION}

La impregnación consiste en hacer penetrar, en el conjunto de poros, microporos y vacíos del hormigón, un material de pequeña viscosidad y, ulteriormente, dejarlo endurecer.

Dos categorías se pueden considerar entre los materiales aplicables:

Los que endurecen por polimerización y aquellos que se solidifican por enfriamiento.

La segunda categoría de productos deben poseer, según Niels Thaulow ("Sulfur impregnated Concrete" Cement and Concrete Research. Vol. 4, págs. 269-277, 1947), las siguientes condiciones: Bajo costo. Abundancia. Punto de fusión entre 100 y $400^{\circ} \mathrm{C}$. Débil viscosidad, en estado líquido para mojar bien el hormigón. Buenas características mecánicas, en estado sólido. Gran inercia química. Débil presión de vapor, en estado sólido. Difícilmente inflamable.

El azufre posee cualidades que corresponden bien a estas condiciones. Su punto de fusión es $120^{\circ} \mathrm{C}$, su viscosidad inferior a 10 centipoises alrededor de $155^{\circ} \mathrm{C}$, su tensión superficial del orden de 55 dinas $/ \mathrm{cm}$.

* Reseña y traducción: P. G. de P. (IETCC). 
Los autores consideraron necesario un estudio experimental de las posibilidades de reacción entre el cemento y el azufre, a pesar de la débil actividad química de este último; por ello han centrado el estudio en la durabilidad del material obtenido.

\section{PROPIEDADES DEL AZUFRE}

Abundante en los terrenos volcánicos. En Francia se obtiene por oxidación del hidrógeno sulfurado contenido en el gas natural de Lacq, que le contiene en un $16 \%$.

Al estado sólido el azufre existe en dos formas cristalinas:

Azufre alfa $\mathrm{S} \%$ rómbico, que a lus új $\mathrm{C}$ se transforma en azufre beta $\mathrm{S} \beta$. A los $120^{\circ} \mathrm{C}$ funde en un líquido amarillo, denominado Azufre landa $\mathrm{S} \lambda$ formado por moléculas cíciicas de ocho átomos. La viscosidad del líquido a los $160^{\circ} \mathrm{C}$ sube extraordinariamente y se forma eì azufre blando o azufre mu $\mathrm{S} \mu$; este aumento de viscosidad es debido a la rotura de las moléculas cíclicas.

Es, pues, importante para la impregnación mantener el baño de azufre fundido a temperatura inferior a $160^{\circ} \mathrm{C}$. La zona de viscosidades aceptables oscila entre 150 y $155^{\circ} \mathrm{C}$.

El azufre presenta una inercia química relativamente grande. A temperatura ambiente reacciona violentamente con el cloro gaseoso.

Principales reacciones del azufre

\begin{tabular}{|c|c|}
\hline Compuestos & Tipes de reacción \\
\hline $\begin{array}{lllllllllllllll}\text { Agua } & \ldots & \ldots & \ldots & \ldots & \ldots & \ldots & \ldots & \ldots & \ldots & \ldots & \ldots & \ldots & \ldots & \ldots\end{array}$ & $\begin{array}{l}\text { Muy lenta por debajo de } 350^{\circ} \mathrm{C} \text {, pero acelerada en pre- } \\
\text { sencia de iones alcalino-térreos. Forma sulfuros, poli- } \\
\text { sulfuros. }\end{array}$ \\
\hline Hidróxidos cálcico y sódico $\mathrm{Ca}(\mathrm{OH})_{2}$ y $\mathrm{NaOH} \ldots$ & $\begin{array}{l}\text { Aceleran las reacciones con el agua. Reaccionan en } \\
\text { caliente. }\end{array}$ \\
\hline $\begin{array}{lllllllllll}\text { Acido } & \text { clorhídrico } & \mathrm{HCl} & \ldots & \ldots & \ldots & \ldots & \ldots & \ldots & \ldots & \ldots\end{array}$ & Ninguna reacción, salvo en presencia de hierro \\
\hline 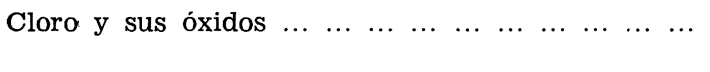 & $\begin{array}{l}\text { Reacción lenta en disolución acuosa, pero muy violenta } \\
\text { en seco. }\end{array}$ \\
\hline $\begin{array}{lllllllllllllll}\text { Hierro } & \ldots & \ldots & \ldots & \ldots & \ldots & \ldots & \ldots & \ldots & \ldots & \ldots & \ldots & \ldots & \ldots & \ldots\end{array}$ & $\begin{array}{l}\text { Acción violenta si el hierro y el azufre están muy di- } \\
\text { vididos e íntimamente mezclados. }\end{array}$ \\
\hline
\end{tabular}

\section{TRABAJOS REALIZADOS EN OTROS LABORATORIOS}

Parece que los primeros ensayos de impregnación con azufre datan de 1925 (R. P. Bacon. VH. Kobev. US Paten 1561767). Calentaban el hormigón a $120^{\circ} \mathrm{C}$ y lo sumergían en un baño de azufre a $125^{\circ} \mathrm{C}$ durante unas horas.

En el Canadá (Malnotra I. ${ }^{\text {er }}$ Inter-Congres on Poliner Concrete. 5-5-1975) impregnaban hormigones de 24 horas secados a $70^{\circ} \mathrm{C}$ durante 24 horas 192 horas. Seguidamente sumergidos en un baño de azufre a $125^{\circ} \mathrm{C}$; durante 2 horas. Sometían el baño y su contenido a un vacío de $15 \mathrm{~mm}$ de mercurio durante $1 / 2$ hora. La riqueza de azufre incorporado alcanzaba el $13 \%$. Las resistencias subían de $714 \mathrm{kp} / \mathrm{cm}^{2}$ iniciales a $1.122 \mathrm{kp} / \mathrm{cm}^{2}$. También aplicaban otro método, sin previo secado, que permitía obtener una resistencia de 683,4 $\mathrm{kp} / \mathrm{cm}^{2}$ a partir de $132,6 \mathrm{kp} / \mathrm{cm}^{2}$.

Niels Thaulow (Cement and Concrete Research., Vol. 4, págs. 269-277 Pergamon Press. Inic. 1974) utilizó cilindros de hormigón, con2 años de edad y resistencia de $653 \mathrm{kp} / \mathrm{cm}^{2}$. La impregnación comprendió un secado a $120^{\circ} \mathrm{C}$, durante 4 días, inmersión en baño de 
azufre a $120 \mathrm{k} 150^{\circ} \mathrm{C}$, vacío durante 1 hora $\mathrm{y}$, después, presión de un gas inerte de $1 / 2$ hora a 2 horas. El material adquiría una tasa de azufre de 8,4 \%, y la compresión alcanzó $1.734 \mathrm{kp} / \mathrm{cm}^{2}$.

En el Japón (Skimura, Y. Murota. T. Niki. K. Sekine. The Cement Association of Japán. Tokyo 1975) con 20 horas de inmersión en azufre fundido la resistencia en flexión crece de 71,4 a $214,2 \mathrm{kp} / \mathrm{cm}^{2}$, y en compresión de 357 a $1.224 \mathrm{kp} / \mathrm{cm}^{2}$.

\section{ENSAYOS REALIZADOS EN EL CERIB}

El CERIB proyectó un programa de ensayos especialmente acentuado en la determinación de los límites o las condiciones de fabricación y de empleo del nuevo material "hormigón impregnado en azufre".

\section{A) Estudio de las diferentes fases del tratamiento}

La experiencia adquirida en el estudio de la impregnación con resinas (plásticos) ha permitido orientar los ensayos dirigidos hacia la impregnación parcial, técnica que no exige un recinto hermético por la ausencia de la fase previa de desgasificación, ni la aplicación de un gas inerte a presión.

Es una técnica mucho más sencilla y económica más viable que la impregnación total. La generalidad de los productos de hormigón manufacturado impone, efectivamente, una geometría que no se podría modificar incluso si se mejorase considerablemente las cualidades intrínsecas del hormigón a causa de una impregnación total.

La técnica de la impregnación parcial comprende las fases siguientes que han sido sistemáticamente mejoradas.

Secado del hormigón. Inmersión en el baño de azufre fundido. Endurecimiento del azufre introducido mediante la refrigeración del hormigón.

\section{1) Modalidades de los ensayos}

Los ensayos se realizaron en probetas de hormigón con las dimensiones $7 \times 7 \times 28 \mathrm{~cm}$.

La composición del hormigón utilizado en la confección de las probetas fue:

Cemento CPA-400: $350 \mathrm{~kg} / \mathrm{m}^{3}$. Arena $0-6: 820 \mathrm{~kg} / \mathrm{m}^{3}$. Gravilla rodada 4-8: $960 \mathrm{~kg} / \mathrm{m}^{3}$. Agua $205 \mathrm{~kg} / \mathrm{m}^{3}$. Relación agua $/$ cemento $=0,6$.

Asentamiento en el cono de Abrams: $3 \mathrm{~cm}$.

\section{2) Influencia del secado del hormigón}

En presencia de iones alcalinos o alcalino-térreos, el agua puede reaccionar con el azufre dando lugar a la formación de tiosulfatos y de polisulfuros los cuales, a largo plazo, se pueden transformar en sulfatos, agentes destructores del hormigón. 
Las experiencias han tenido como fin determinar en particular el grado necesario de secado que evite la formación de sulfatos.

Después de su fabricación, las probetas se conservan en cámara húmeda y, a continua.ción se secan, unas a peso constante a $140^{\circ} \mathrm{C}$ y otras durante 4 horas en estufa a $140^{\circ} \mathrm{C}$ (contenido de agua $1,2 \%$ ). Otra serie de probetas se mantiene durante 24 horas al aire libre (contenido de agua 6,7 \%). Estas son, entonces, sumergidas durante 3 horas en un baño de azufre cuya temperatura se mantiene a $150 \pm 5^{\circ} \mathrm{C}$. Después de las 3 horas, se retiran del baño las probetas, y escurridas se sumergen durante algunos segundos en agua fría para solidificar el azufre superficial; a continuación se dejan enfriar al aire libre.

En la tabla siguiente se indican los resultados obtenidos en función de la cantidad de agua contenida en el hormigón antes de la impregnación.

\begin{tabular}{lcccc}
\hline Contenido de agua & $\begin{array}{c}\text { Testigos no } \\
\text { impregnados } \\
\text { (3 días) }\end{array}$ & $6,7 \%$ & $1,2 \%$ & 0 \\
Valor de la impregnación (en masa) & - & $5,2 \%$ & $6,7 \%$ & $7,4 \%$ \\
$\begin{array}{l}\text { Profundidad de la impregnación } \\
\text { (en mm) }\end{array}$ & - & 9 & 15 & 18 \\
$\begin{array}{l}\text { Resistencia a flexión } \\
\text { Resistencia a compresión }\end{array}$ & $25 \mathrm{kp} / \mathrm{cm}^{2}$ & 30 & 43 & 50 \\
\hline
\end{tabular}

La zona impregnada de las probetas no desecadas presenta una coloración verde muy fuerte. En el caso de las probetas secadas duran 'e 4 horas, la coloración verde también está presente, pero más ligera. Estas coloraciones son características de los polisulfuros.

Las observaciones realizadas con el microscopio electrónico de barrido muestran, en el hormigón no desecado totalmente, la presencia de cristales de sulfoaluminato cálcico. Estos cristales están ausentes en los casos del hormigón impregnado después de la desecación total. Estas observaciones podrían explicar los malos resultados obtenidos con la impregnación en experiencias anteriores.

\section{3) Influencia del tiempo de impregnación}

La cantidad de azufre que penetra en el hormigón y la profundidad de la penetración son funciones del tiempo que permanecen las probetas sumergidas en el baño de azurfe, donde, después del secado, se las sumerge en el azufre líquido manteniéndose a $150^{\circ} \mathrm{C}$.

\begin{tabular}{lcccc}
\hline & $\begin{array}{c}\text { Riqueza } \\
\text { de azufre } \\
(\%)\end{array}$ & $\begin{array}{c}\text { Profundidad } \\
\text { de impregnación } \\
(\mathbf{m m})\end{array}$ & $\begin{array}{c}\text { Resistencia } \\
\mathbf{a} \text { flexión } \\
\left(\mathbf{k p} / \mathbf{c m}^{2}\right)\end{array}$ & $\begin{array}{c}\text { Resistencia } \\
\mathbf{a} \text { compresión } \\
\left(\mathbf{k p} / \mathbf{c m}^{2}\right)\end{array}$ \\
\hline Tratamiento $a$ & 5,3 & 12 & 54 & 607 \\
Tratamiento $b$ & 5,5 & 13 & 55 & 637 \\
Tratamiento $c$ & 5,2 & 12,5 & 52 & 663 \\
Tratamiento $d$ & 4,7 & 13 & 46 & 647 \\
Medias & 5,2 & 12 & 52 & 635 \\
\hline
\end{tabular}

Las débiles variaciones observadas parecen indicar que este parámetro no tiene efecto sobre los resultados de la impregnación.

\section{B) Estudio de la influencia de la textura del hormigón}

La textura del hormigón varía, entre otras cosas, con la relación agua/cemento y con la edad, en particular en función del estado de las reacciones de hidratación y el grado de carbonatación. 


\section{1) Influencia de la relación agua/cemento}

Las resistencias a la compresión del hormigón impregnado, mayores que la de los testigos, evolucionan paralelamente unas de otras en sentido descendente al aumentar la relación agua/cemento desde 0,4 a 0,8 .

2) Influencia de la edad del hormigón: Resistencia a compresión

\begin{tabular}{|c|c|c|c|c|c|}
\hline Edad del hormigón: días $\ldots \ldots \ldots \ldots$ & 1 & 3 & 5 & 7 & 28 \\
\hline $\begin{array}{llllllllll}\operatorname{Impregnadd} & \ldots & \ldots & \ldots & \ldots & \ldots & \ldots & \ldots & \ldots & \ldots\end{array}$ & 525 & 530 & 570 & 600 & 635 \\
\hline $\begin{array}{lllllllll}\text { No impregnado } & \ldots & \ldots & \ldots & \ldots & \ldots & \ldots & \ldots & \ldots\end{array}$ & - & 220 & 355 & 410 & 425 \\
\hline
\end{tabular}

La cantidad de azufre y la profundidad alcanzada disminuyen cuando la edad varía de 0 a 10 días, y a partir de esta edad no sufren variación.

De una manera semejante entre sí, evolucionæa con el tiempo de inmersión: la profundidad, la cantidad de azufre introducido, la resistencia a la compresión y la resistencia a la flexión. Todas estas funciones son crecientes hasta aproximadamente las 6 horas y tienden a estabilizarse a las 8 horas.

\section{INFLUENCIA DE LA TEMPERATURA DEL HORMIGON AL SUMERGIRLO EN EL BAÑ O}

El baño de azufre se mantiene a $150^{\circ} \pm 5^{\circ} \mathrm{C}$. El hormigón al terminar el secado alcanza la temperatura de $140^{\circ} \mathrm{C}$.

Se realizaron ensayos para comprobar la influencia que tendría la diferencia de temperatura entre el hormigón y el baño, como ocurre en la impregnación con polímeros.

Después del secado se mantuvieron probetas a $70^{\circ} \mathrm{C}-100^{\circ} \mathrm{C}-120^{\circ} \mathrm{C}$ y $140^{\circ} \mathrm{C}$. Estas temperaturas se eligieron teniendo en cuenta las velocidades de transformación del azufre $\alpha$ en azufre $\beta$ en función de la temperatura. La primera temperatura es inferior a la transformación del azufre $\alpha$ en azufre $\beta$. La segunda está en el interior del dominio de estabilidad del azufre $\beta$. La textura corresponde al punto de fusión del azufre $\beta$, y la última está situada en el dominio de estabilidad del azufre liquido $\lambda$.

\begin{tabular}{lccccc}
\hline $\begin{array}{c}\text { Temperatura del } \\
\text { hormigón...antes } \\
\text { de ser sumergido }\end{array}$ & $\begin{array}{c}\text { Testigos no } \\
\text { impregnados }\end{array}$ & $\mathbf{7 0 ^ { \circ } \mathbf { C }}$ & $\mathbf{1 0 0 ^ { \circ } \mathbf { C }}$ & $\mathbf{1 2 0} \mathbf{C}$ & $\mathbf{1 4 0 ^ { \circ } \mathbf { C }}$ \\
\hline Cantidad impregnada & & 4,2 & 4,4 & 4,8 & 4,7 \\
Profundidad (mm) & $32 \mathrm{kp} / \mathrm{cm}^{2}$ & 46 & 10 & 11 & 11 \\
Resistencia a tracción & $360 \mathrm{kp} / \mathrm{cm}^{2}$ & 530 & 610 & 600 & 58 \\
Resistencia a compresión & & 50 & 46 & 580 \\
\hline
\end{tabular}

Parece que la temperatura del hormigón no tiene influencia en el resultado final de la impregnación.

Influencia del modo de endurecimiento del azufre.

La velocidad de enfriamiento del azufre tiene una influencia sobre la cinética de la transformación del azufre $\beta$ en azufre $\alpha$. 
La influencia de la velocidad de enfriamiento del hormigón impregnado de azufre sobre las características obtenidas se estudió según cuatro modos de enfriamiento.

a) Inmersión durante 5 minutos en un baño de agua con hielo a $0^{\circ} \mathrm{C}$.

b) Inmersión durante 10 a 15 segundos en agua a $20^{\circ} \mathrm{C}$ seguida de enfriamiento al aire libre.

c) Enfriamiento al aire libre.

d) Enfriamiento muy lento en la estufa cuya temperatura decrecía lentamente.

Las débiles variaciones obtenidas parecen indicar que este parámetro no tiene efecto sobre los resultados de la impregnación.

\section{C) Estudio del efecto beneficioso de la impregnación con azufre}

1) Características mecánicas

Las resistencias obtenidas son evidentemente función de la cantidad y la profundidad de la impregnación; aumentan linealmente desde los 5 a los $20 \mathrm{~mm}$ de profundidad.

\section{2) Módulo de deformación}

La impregnación con azufre aumenta los módulos de deformación sin que este incremento aparezca ligado a la cantidad fijada.

\section{3) Resistencia al desgaste}

Se ha podido comprobar un incremento en la resistencia al desgaste en la impregnación con azufre, pero es sensiblemente inferior al obtenido con la impregnación con plásticos.

\section{4) Absorción al gua}

La impregnación de los hormigones tiene por consecuencia que, sea superficial o no, limita los fenómenos de orden capilar en el hormigón.

La absorción de agua y las variaciones dimensionales deben disminuir, y la resistencia a la helada y deshielo se mejoran notablemente.

\section{D) Estudio de la durabilidad}

Las características obtenidas después de la impregnación con azufre son muy superiores a las del hormigón no impregnado. La impregnación con azufre parece una técnica interesante para mejorar las cualidades del hormigón, especialmente las superficiales.

Pero en ciertas condiciones, el azufre puede reaccionar con el hormigón dando lugar a una pérdida notable de resistencias. Este fenómeno se ha comprobado cuando el hormigón está mal secado; las reacciones son muy rápidas pues se producen a la temperatura elevada del baño de azufre líquido.

50 
Dos tipos de reacciones se pueden producir. La primera según el esquema:

S elemental $\stackrel{\text { Alcalino térreo }+ \text { agua }}{\longrightarrow}$ sulfuros, polisulfuros $+\stackrel{\text { tiosulfatos }}{\longrightarrow}$ sulfatos $\stackrel{\text { aluminato tricálcico }}{\longrightarrow}$ sulfoaluminatos expansivos.

Estas reacciones producen la destrucción del hormigón por expansión.

La segunda se debe a los iones $\mathrm{Na}^{+}$

$$
2 \mathrm{NaOH}+\mathrm{S} \stackrel{\text { agua }}{\longrightarrow} \mathrm{Na}_{2} \mathrm{~S} \stackrel{\mathrm{S}}{\longrightarrow} \mathrm{Na}_{2} \mathrm{~S}_{2} \longrightarrow \mathrm{Na}_{2} \mathrm{~S}_{\mathrm{x}} \text { (polisulfuros) }
$$

Se produce una extracción del azufre sin destrucción del hormigón, pero la resistencia del material puede disminuir hasta alcanzar el límite del hormigón antes de la impregnación.

Los ensayos en curso en el CERIB tienen por objeto determinar la velocidad de esas, reacciones y precisar su importancia.

Cierto número de probetas preparadas con azufre puro, azufre mezclado íntimamente con cemento, un mortero impregnado y un mortero testigo, se han sumergido en baños de los agresivos tales como: ácido clorhídrico ( $\mathrm{pH} 1)$, ácido acético ( $\mathrm{pH} 3,5)$, agua, lejía de sosa ( $\mathrm{pH} 13)$, cal sodada ( $\mathrm{pH} 12$ ), cal sodada y alúmina, cal sodada y cloruro sódico.

La tabla siguiente reúne los resultados obtenidos a los 3 meses.

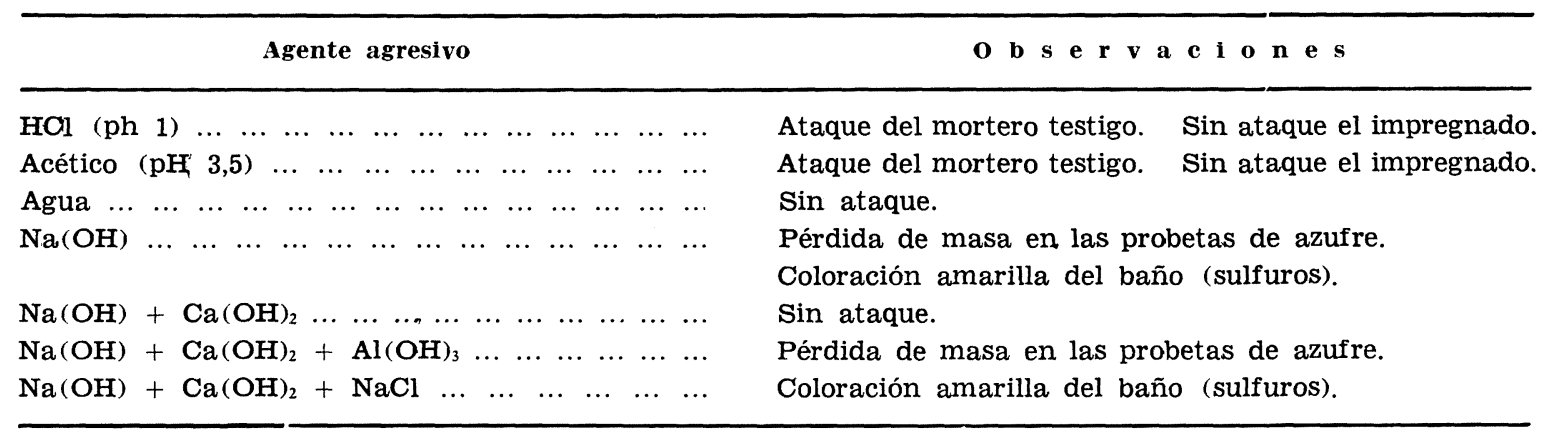

Parece que el ataque podría tener lugar rápidamente en presencia de iones $\mathrm{Na}^{+}$procedentes del agresor externo.

Las observaciones sistemáticas con el microscopio electrónico de barrido sobre secciones de las probetas rotas a la edad de 3 meses no han acusado la presencia de sulfoaluminato expansivo.

El problema mayor reside en la durabilidad del nuevo material y solamente los ensayos a largo plazo permitirán precisar si las mayorías observadas perduran con el tiempo. 